\title{
A Comparison of Ligase Chain Reaction to Polymerase Chain Reaction in the Detection of Chlamydia trachomatis Endocervical Infections
}

\author{
J.D. Davis, ${ }^{1 *}$ P.K. Riley, ${ }^{1}$ C.W. Peters, ${ }^{2}$ and K.H. Rand ${ }^{3}$ \\ ${ }^{1}$ Department of Obstetrics and Gynecology, University of Florida College of Medicine, \\ Gainesville, $F L$ \\ ${ }^{2}$ Department of Emergency Medicine, University of Florida College of Medicine, Gainesville, FL \\ ${ }^{3}$ Department of Pathology, Immunology and Laboratory Medicine, University of Florida College of \\ Medicine, Gainesville, FL
}

\begin{abstract}
Objective: To compare the reliability of ligase chain reaction (LCR) to polymerase chain reaction (PCR) in detecting Chlamydia trachomatis endocervical infections.

Methods: We conducted a prospective study of $\mathbf{4 8 6}$ patients at risk for chlamydial infection of the endocervix. We obtained two endocervical specimens from each patient and used LCR and PCR to detect $C$. trachomatis. Discrepant results between the two techniques were resolved by repeat testing and by testing for the major outer membrane protein (MOMP) gene, if necessary. We determined the sensitivity, specificity, positive predictive value, and negative predictive value for each test, using concordant results or MOMP gene results as the "gold standard."

Results: Of the 486 patients, $42(8.6 \%)$ had evidence of $C$. trachomatis infection after resolution of discrepant results. Of the 42 true positive specimens, 41 were positive by initial LCR and 38 were positive by initial PCR. Of the 444 true negative specimens, none had a positive initial LCR result, while 2 had a positive initial PCR test. Therefore, compared to the gold standard, LCR had a sensitivity of $97.6 \%$ and specificity of $100 \%$, while PCR had a sensitivity of $90 \%$ and a specificity of 99.5\% . The positive and negative predictive values of LCR were $100 \%$ and $99.8 \%$, respectively. PCR had a positive predictive value of $95 \%$ and a negative predictive value of $99.1 \%$. The difference in sensitivity of $L C R$ versus PCR was not statistically significant $(P=.125)$.

Conclusion: LCR and PCR perform equally well in detecting C. trachomatis endocervical infections. Infect. Dis. Obstet. Gynecol. 6:57-60, 1998. ๑ 1998 Wiley-Liss, Inc.
\end{abstract}

KEY WORDS

chlamydial infection; endocervix; DNA amplification techniques

C ulture in mammalian cell lines has been considered the "gold standard" for diagnosing Chlamydia trachomatis; however, this method is labor intensive, time consuming, and expensive. Non-culture methods, including direct fluorescent antibody (DFA) testing and enzyme immunoassays, have been employed but lack the sensitivity and specificity of tissue culture. ${ }^{1}$ More recently, DNA amplification techniques, including the polymerase chain reaction (PCR)-based Amplicor $G$. trachomatis Test (Roche Molecular Systems, Branchburg, NJ) and the ligase chain reaction (LCR)-based LCx Probe System (Abbott Laboratories, Chicago, IL), have been used to diagnose

Grant sponsor: Abbott Laboratories, Chicago, Illinois. Presented at the 45th Annual Clinical Meeting of the American College of Obstetricians and Gynecologists, Las Vegas, Nevada, April 26-30, 1997.

*Correspondence to Dr. John D. Davis, Director, Division of Gynecology, Department of Obstetrics and Gynecology, University of Florida College of Medicine, PO Box 100294, Room M-302E, Gainesville, FL 32610-0294. E-Mail: davisjd@obgyn.med.ufl.edu 
urogenital chlamydial infections with improved sensitivity. ${ }^{2,3}$

To our knowledge, two studies have been published comparing LCR to PCR in the detection of chlamydial infections of the urogenital tract. In the first study, de Barbeyrac et al. ${ }^{4}$ found LCR to be more sensitive than PCR in the diagnosis of endocervical and urethral chlamydial infections; the difference in sensitivity was not statistically significant. In the second study, Pasternack et al. ${ }^{5}$ likewise could find no statistically significant difference between LCR and PCR; however, they believed the manual PCR was superior to LGR, because only the manual PGR correctly identified all chlamydia-positive patients. The purpose of our study was to determine if either LCR or PCR was superior in detecting $C$. trachomatis endocervical infections.

\section{SUBJECTS AND METHODS}

Between April 1 and July 4, 1996, we obtained two endocervical specimens from 486 women seen at the University of Florida Shands Hospital who were at risk for chlamydial infection. Specimens were obtained from new obstetric patients; gynecologic patients with pelvic pain, mucopurulent cervical discharge, or other signs or symptoms possibly attributable to chlamydia infection; and all reproductive-aged women seen in the Emergency Department regardless of their chief reason for seeking treatment. We conducted this study under a protocol approved by the Institutional Review Board.

To obtain specimens, examiners first removed any cervical discharge with a large cotton swab. They then sampled the endocervical canal sequentially with two Dacron swabs, rotating each swab for 15-30 seconds to ensure adequate sampling. They placed and left the swab for LCR testing into the LCR transport media. The examiners vigorously swirled the swab for PCR testing in the PCR transport media, expressed excess fluid, and removed the swab in accordance with the manufacturer's instructions. The specimens were kept at room temperature and transported to the laboratory within 12 hours of collection and kept refrigerated for not more than 48 hours prior to testing. Laboratory personnel were blinded to the LCR and PCR results for each matched pair until the final result for each method was determined. After test- ing, specimens were held frozen at $-70^{\circ} \mathrm{C}$ in case further studies needed to be performed.

The LCx Probe System uses four primers with specificity for the $C$. trachomatis cryptic plasmid. Laboratory technicians performed this test after direct training from the manufacturer using methods previously described. ${ }^{3}$ Briefly, the specimen was first heat denatured at $97^{\circ} \mathrm{C}$ for 15 minutes and 100 $\mu \mathrm{l}$ was added to amplification vials containing all reagents for amplification. The amplification was carried out in a thermocycler for 40 cycles, and the entire run was transferred to the analyzer which adds all reagents for the detection of the amplified product. The amplification and detection steps were performed in a physically separate area from the setup of new specimens. All test runs included appropriate calibrators, together with positive and negative controls provided by the manufacturer.

PCR was performed according to the instructions from the manufacturer as previously described. ${ }^{2}$ In this procedure, $1 \mathrm{ml}$ of Specimen Diluent was added to the patient specimen and $50 \mu \mathrm{l}$ of the mixture was added to the PCR tube containing the PCR Master Mix. After 30 cycles of thermal amplification in the presence of biotin labeled primers also directed towards the $C$. trachomatis cryptic plasmid, the reaction mixture was transferred to a microtiter plate coated with a DNA capture probe. The amplified product was then detected with the addition of avidin-horseradish peroxidase in an ELISA format. Thermocycling was carried out with Uracil-N-glycosylase to prevent carryover contamination. As specified by the manufacturer, one positive control and one negative control tested in triplicate were included with each test run.

All specimens testing within the equivocal range for either method were repeated twice. None of the PCR results were equivocal. Only two of the LCR results were in the equivocal range. On repeat testing, one specimen was positive and one was negative.

Any specimen that tested positive by one method but not by the other was tested again by both methods, and all such discrepant specimens were sent to the Roche and Abbott laboratories under code for repeat testing and resolution using primers for the major outer membrane protein (MOMP) gene, if necessary. We calculated the sensitivity, specificity, and positive and negative pre- 
TABLE I. Resolution of discrepant results at Abbott and Roche Laboratories

\begin{tabular}{|c|c|c|c|c|c|}
\hline $\begin{array}{l}\text { Case } \\
\text { number }\end{array}$ & $\begin{array}{c}\text { Initial } \\
\text { LCR result }\end{array}$ & $\begin{array}{c}\text { Initial } \\
\text { PCR result }\end{array}$ & $\begin{array}{l}\text { Resolved LCR } \\
\text { result (Abbott) }\end{array}$ & $\begin{array}{l}\text { Resolved PCR } \\
\text { result (Roche) }\end{array}$ & $\begin{array}{c}\text { Final } \\
\text { diagnosis }\end{array}$ \\
\hline 1 & + & - & + & + & + \\
\hline 2 & + & - & + & + & + \\
\hline 3 & + & - & + & + & + \\
\hline 4 & + & - & + & + & + \\
\hline 5 & - & + & + & Not done* & + \\
\hline 6 & - & + & - & - & - \\
\hline 7 & - & + & - & - & - \\
\hline
\end{tabular}

*The remaining PCR specimen was accidentally discarded.

dictive values for both tests using concordant results on initial or repeat testing or MOMP gene results for persistently discordant tests as the gold standard. We then performed an exact form of the McNemar test to determine if the difference in sensitivities of the two tests was significant.

\section{RESULTS}

Of the 486 patients, 442 tested negative initially by both tests, while 37 patients were positive initially by both LGR and PGR. Seven patients had discordant initial LCR and PGR results (Table 1). Four patients were positive by LCR and negative by PCR (cases 1-4). Repeat testing in our laboratory confirmed these results. Upon duplicate testing on undiluted specimens at the outside laboratories, all four PCR specimens tested positive; the LCR results remained unchanged. Three patients were negative by LGR and positive by PGR (cases $5-7$ ). Repeat testing in our laboratory confirmed the initial results. Two of these patients (numbers 6 and 7) had both negative LCR and PCR tests on undiluted specimens at the outside laboratories (initial false-positive PCR tests). The remaining patient (number 5) tested positive by LCR when the test was repeated on an undiluted specimen (initial false negative LCR). Testing for the MOMP gene was not required to resolve discrepancies on any specimens.

Of the 486 patients, $42(8.6 \%)$ had evidence of C. trachomatis infection after resolution of discrepant results. Of the 42 true positive specimens, 41 tested positive by initial LCR and 38 tested positive by initial PCR. Of the 444 specimens negative by both methods on initial or repeat testing, none had a positive initial LCR result, while two had a positive initial PCR result. Therefore, using the resolved results as the gold standard, LGR had a sensitivity and specificity of $97.6 \%$ and $100 \%$, respectively, while PGR had a sensitivity of $90 \%$ and a specificity of $99.5 \%$. The positive and negative predictive values of LGR were $100 \%$ and $99.8 \%$, respectively. PCR had a positive predictive value of $95 \%$ and a negative predictive value of $99.1 \%$. The difference in sensitivity of LCR versus PCR was not statistically significant $(P=.125)$.

\section{DISCUSSION}

LCR and PGR techniques both utilize DNA amplification technology, and both methods are more sensitive in diagnosing $C$. trachomatis endocervicitis than older methods, such as culture, DFA, or ELISA. In this head to head comparison using endocervical specimens, the original LCR result proved to be correct in six of the seven discordant pairs while the original PCR result was correct in only one pair. The difference in performance of LGR versus PCR was not statistically significant.

In a study of over 2,000 patients, Schachter et al. compared LCR to cell culture for the diagnosis of $C$. trachomatis endocervicitis using endocervical specimens and found the sensitivity of LCR was $94 \%$ versus $65 \%$ for culture. ${ }^{1}$ These authors concluded LCR was a highly sensitive method for detecting $C$. trachomatis endocervicitis and called for a direct comparison between LGR and PGR to determine which test was better. The first such study was published by de Barbeyrac et al., ${ }^{4}$ who studied 134 women from a low-risk population and 84 women and 62 men from a high-risk population. In the female group, the authors compared the performance of LCR, PCR, and tissue culture using endocervical specimens. A specimen was considered truly positive if the culture was positive, if the LCR and PCR were both positive, or if the MOMP gene was positive when the LCR and PCR results 
were discordant. A specimen was truly negative if the culture and either the LCR or PCR were negative. The sensitivity of LCR was $95.2 \%$ versus $80.9 \%$ for both PCR and culture; the specificity of both LCR and PCR was 99.6\%. The difference in sensitivity of LCR versus PCR was not statistically significant. The authors concluded LCR was a good alternative to PCR in the detection of chlamydial infections.

Pasternack et al. ${ }^{5}$ recently compared the results of LCR and PCR on first void urine specimens to tissue culture on endocervical specimens in the detection of $G$. trachomatis infections in 442 female patients. Positive culture, positive LGR and PGR results, and a positive MOMP gene test when the LCR and PCR results were discordant were considered truly positive. In their study, PCR had a sensitivity of $100 \%$ and specificity of $99.7 \%$, while the sensitivity and specificity of LCR was $94 \%$ and $100 \%$, respectively. Again, the difference in sensitivity between the two tests was not statistically significant. However, the authors believed the manual PCR was better than LCR because the manual PCR detected all chlamydia-positive patients.

As with the above reports, our study did not demonstrate a statistically significant difference in the performance of LCR versus PCR. However, after resolution of the discordant specimens, the original LCR result proved to be correct in six of the seven discordant pairs while the initial PCR result was correct only once. In our study, there was a single false-negative LCR and four false-negative PCR results. These false-negative results may have been caused by ligase or polymerase inhibitors, a problem to which both DNA amplification tests are susceptible. ${ }^{1,6}$ We cannot confirm this since we did not perform any specific tests to determine if inhibiting factors were present. Whether LCR is possibly less sensitive to inhibitors must await further study.

A limitation of our study is that we did not obtain specimens for confirmatory tissue culture. Therefore, it is possible that some of our concordant pairs were both falsely positive or both falsely negative and our calculated sensitivities and specificities are overestimates. This would be a serious drawback to our study if our purpose was to determine the exact sensitivity and specificity of LCR and PCR. However, our purpose was to directly compare the relative performance of two tests which have already been shown to be more sensitive and nearly as specific as tissue culture. DNA amplification tests are reported to be greater than $90 \%$ sensitive and greater than $99 \%$ specific, while tissue culture is typically $75-85 \%$ sensitive and considered $100 \%$ specific. ${ }^{3-7}$ While our calculated sensitivities and specificities may be overestimates of the true values for both LGR and PGR, the number and nature of the discordant pairs are unaffected. Therefore, even though we did not obtain confirmatory tissue cultures, the relative performance of LCR versus PCR, and the results of our study, are unchanged.

\section{REFERENCES}

1. Schachter J, Stamm WE, Quinn TC, Andrews WW, Burczak JD, Lee HH: Ligase chain reaction to detect Chlamydia trachomatis infection of the cervix. J Clin Microbiol 32:2540-2543, 1994.

2. Loeffelholtz MJ, Lewinski CA, Silver SR, Purohit AP, Herman SA, Buonagurio DA, Dragon EA: Detection of Chlamydia trachomatis in endocervical specimens by polymerase chain reaction. J Clin Microbiol 30:28472851, 1992.

3. Dille BJ, Butzen CG, Birkenmeyer LG: Amplification of Chlamydia trachomatis DNA by ligase chain reaction. J Clin Microbiol 31:729-731, 1993.

4. de Barbeyrac B, Rodriguez P, Dutilh B, Le Roux P, Bebear C: Detection of Chlamydia trachomatis by ligase chain reaction compared with polymerase chain reaction and cell culture in urogenital specimens. Genitourin Med 71:382-386, 1995.

5. Pasternack R, Vuorinen P, Pitkajarvi T, Koskela M, Miettinen A: Comparison of manual Amplicor PCR, Cobas Amplicor PCR, and LCx assays for detection of Chlamydia trachomatis infection in women by using urine specimens. J Clin Microbiol 35:402-405, 1997.

6. Verkooyen RP, Luijendijk A, Huisman WM, Goessens WHF, Kluytmans JAJW, van Rijsoort-Vos JH, Verbrugh HA: Detection of PCR inhibitors in cervical specimens by using the AMPLICOR Chlamydia trachomatis assay. J Clin Microbiol 34:3072-3074, 1996. 


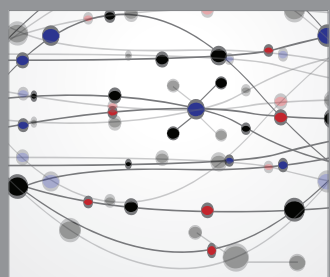

The Scientific World Journal
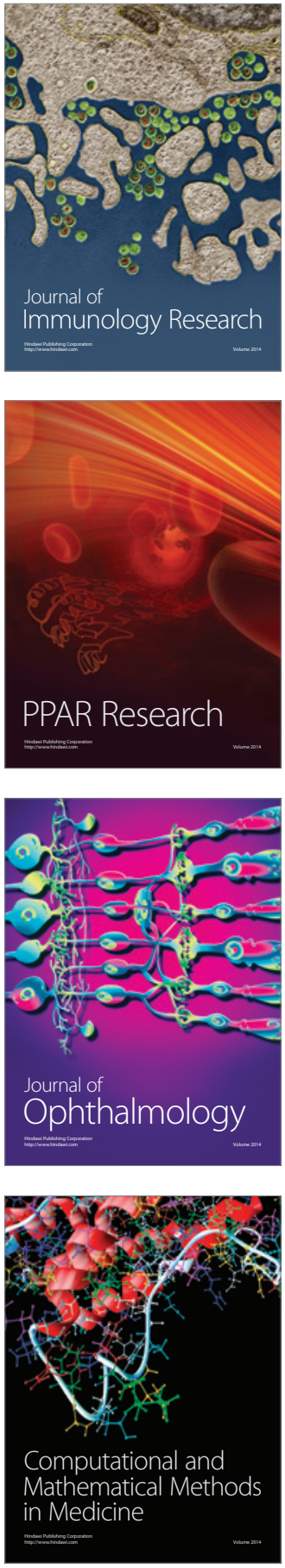

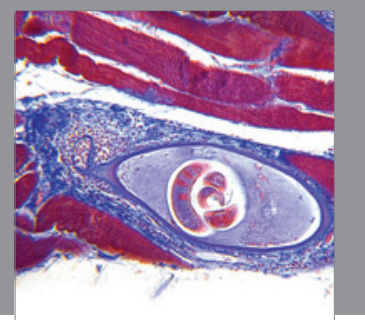

Gastroenterology

Research and Practice
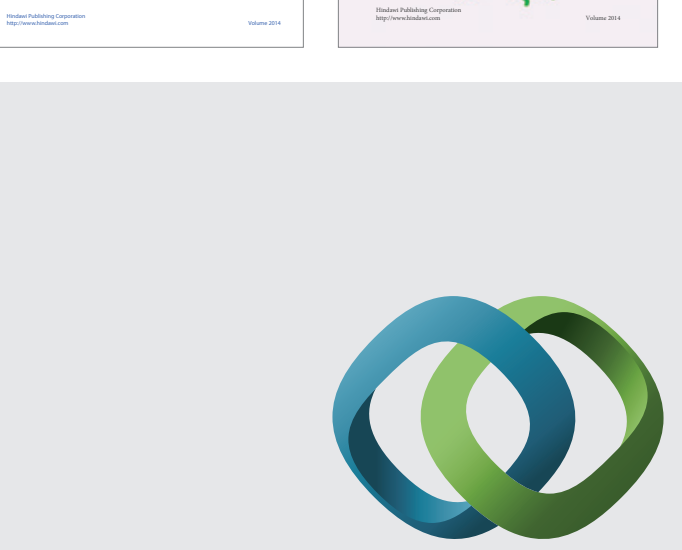

\section{Hindawi}

Submit your manuscripts at

http://www.hindawi.com
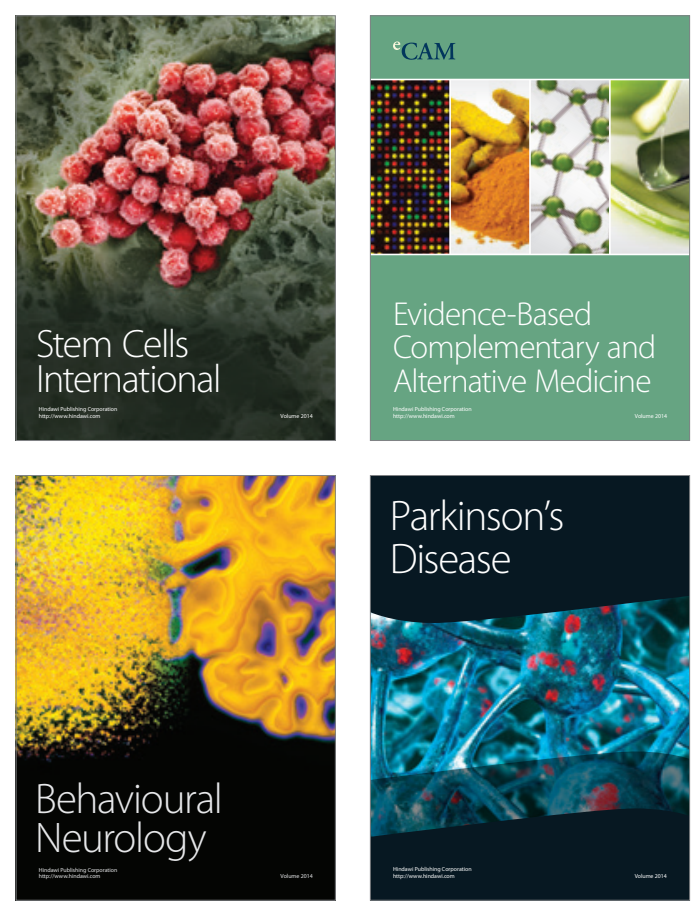

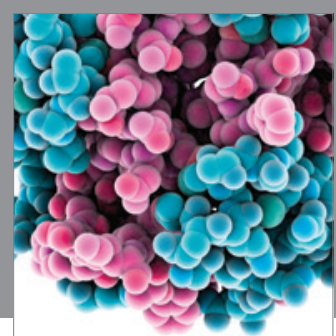

Journal of
Diabetes Research

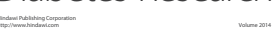

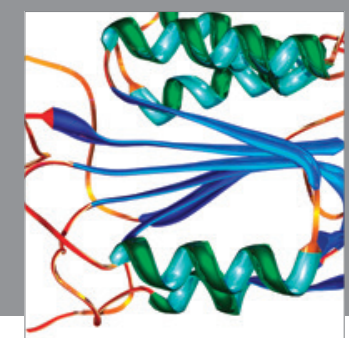

Disease Markers
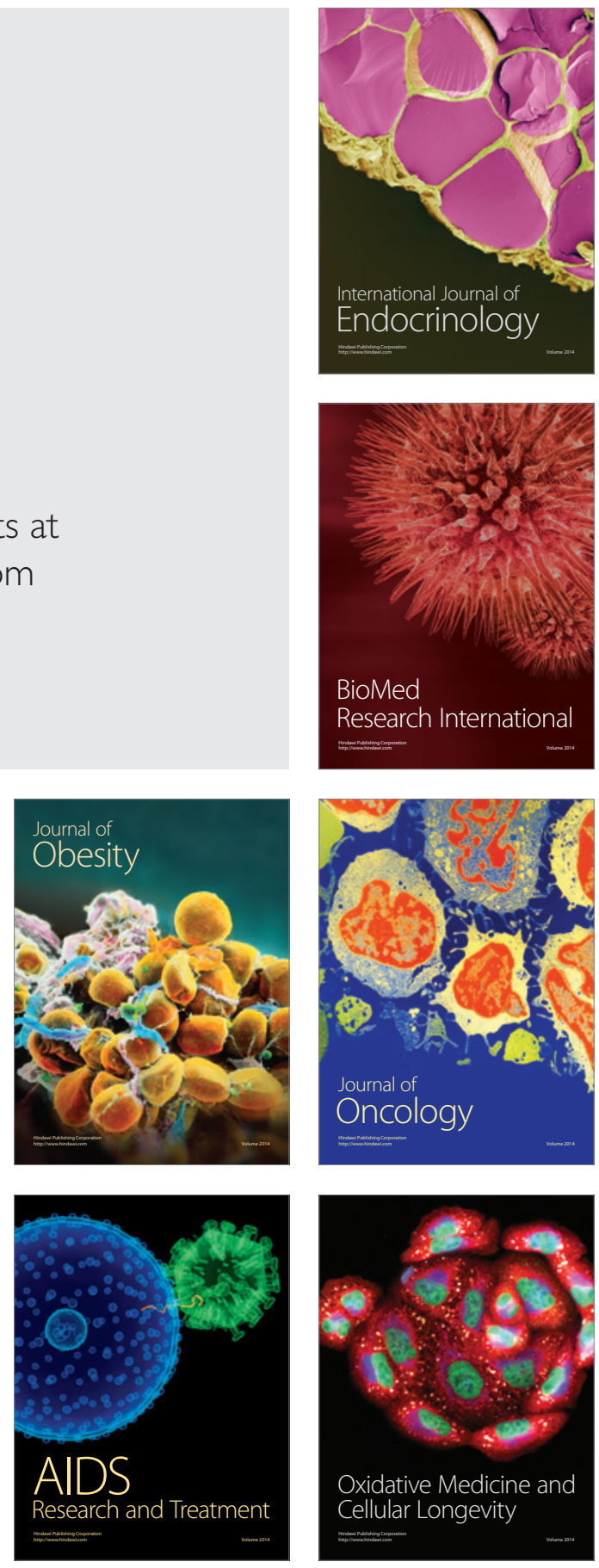